\title{
Hemorrhagic Breakdown of the Cerebral Bridge: A Report of 5 Observations at Lomé Teaching Hospital, Togo
}

\author{
Komi Assogba ${ }^{1,}$, , Michel Faustin Tassa-Kayem ${ }^{1}$, Kossivi Martin Apetse ${ }^{1}$, Damelan Kombate ${ }^{1}$, \\ Jean Joel Tajeuna Dongmo", Josué Euberma Diatewa', Komi Agbotsou ${ }^{1}$, Abdullah Blakime ${ }^{1}$, \\ Veronique Afiwa Agbobli ${ }^{2}$, Kolou Dassa ${ }^{2}$, Koffi Agnon Ayélola Balogou ${ }^{1}$ \\ ${ }^{1}$ Neurology Department, Campus University Teaching Hospital, University of Lomé, Lomé, Togo \\ ${ }^{2}$ Pychology Unit, Campus University Teaching Hospital, University of Lomé, Lomé, Togo
}

Email address:

chocob400@gmail.com (K. Assogba),seraphinassogba@hotmail.com(K. Assogba)

${ }^{*}$ Corresponding author

\section{To cite this article:}

Komi Assogba, Michel Faustin Tassa-Kayem, Kossivi Martin Apetse, Damelan Kombate, Jean Joel Tajeuna Dongmo, Josué Euberma Diatewa, Komi Agbotsou, Abdullah Blakime, Veronique Afiwa Agbobli, Kolou Dassa, Koffi Agnon Ayélola Balogou. Hemorrhagic Breakdown of the Cerebral Bridge: A Report of 5 Observations at Lomé Teaching Hospital, Togo. Clinical Neurology and Neuroscience. Vol. 4, No. 1, 2020, pp. 11-17. doi: 10.11648/j.cnn.20200401.13

Received: February 13, 2019; Accepted: July 4, 2019; Published: Febraury 10, 2020

\begin{abstract}
Introduction: Intracerebral hemorrhage is a major public health problem in Africa. The care is well codified in developed nations but the medical outcome and the technical platform remains a thorny challenge in African countries. It is responsible for a heavy handicap and a high mortality. The corpus callosum is an anatomical structure that connects the two cerebral hemispheres and control neuropsychological behavior. The objective was to determine the epidemiological and clinical characteristics of hematomas located in the corpus callosum. Clinical presentation: The stuy was carried out in the neurlogical department of the big tertiary hospital of the country. The study had reported five cases of hematoma of the corpus callosum. That has represented $0.3 \%$ of hemorrhagic strokes in the service. The average age was 33.4 years old with four females. Clinical symptoms in the acute phase were dominated by speech disorders, intracranial hypertension and meningeal signs. In the subacute phase, all patients had neuropsychological symptoms. These disorders included aphasia, left tactile anomia, ideomotor and diagonostic apraxia, and mood and behavior disorders in two-third of cases. The brain scan was performed with an average delay of 6 days. In the cerebral scan, the posterior part of the corpus callosum was the initial seat of the hematomas observed in all cases. Cerebrovascular risk factors were high blood pressure in all cases and alcoholism in two cases. The factor of poor prognosis was the association with meningeal contamination. The evolution was favorable in all five cases. Conclusion: This work brings out that hematoma of the corpus callosum is rare but still a serious disease with a misleading semiology dominated by neuropsychological disorders. The early management is the best practice to preserve functional autonomy.
\end{abstract}

Keywords: Brain Hematoma, Corpus Callosum, Neuropsychological Disorders, Africa

\section{Introduction}

Stroke is a global health challenge regarding its motor and neurosensory disabilities and its heavy mortality $[1,2]$. Strokes represent the second leading cause of dementia and the first leading cause of acquired physical handicap [3, 4]. The frequency and prognosis of stroke is different and depended on the subtype. Haemorrhagic stroke, $38.5 \%$, are relatively less frequent but associated with high mortality more than arteriovenous infarcts $[5,6]$. The localization of a haemorrhagic event in the corpus callosum is a rare radioclinical condition. The corpus callosum is the main white matter tracts, a pathway that connecting the two hemispheres as a cerebral bridge. It divided into four distinct segments, consists of four each portions (rostrum, genu, trunk, splenium), from which connects different brain areas (figure 1) [7, 8]. 


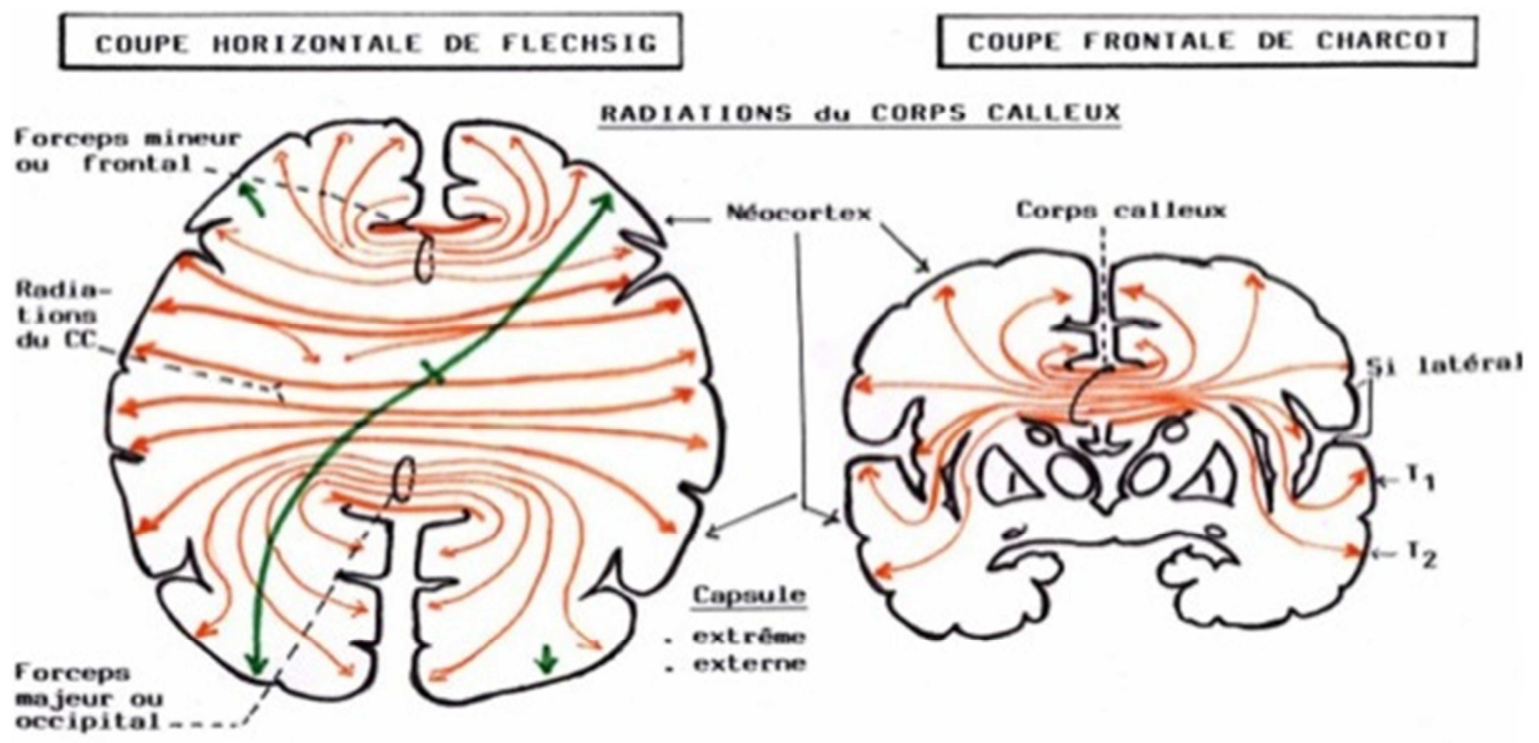

Figure 1. Horizotal section of corpus callosum.

These connections participle in the array of cognitive functions including language, abstract reasoning, integration of complex sensory information between the two hemispheres, and thereby facilitating the integration of motor and sensory information from the two sides of the body [9, 10]. The consequence of the rupture of this bridge is neuropsychological, acute psychosis, sensitive and motor impairement or symptoms [11, 12]. The corpus callosum is necessary for bimanual coordination as well as providing interhemispheric inhibition during unimanual tasks $[10,13]$. Lesions in the corpus callosum have also been linked to gait disturbances including step heights and cadence reduced, and wide base of support $[14,15]$. The objective was to describe the epidemiological, clinical and evolutive aspects of 5 patients suffering from the corpus callosum hemorrhage.

\section{Clinical Cases}

\subsection{Case 1}

A 42-year-old woman, right-handed, trader and married, was admitted to the neurology department for a sudden alteration of consciousness that occurred two hours before the admission. The alteration of consciousness was preceded twelve hours earlier by unusual headaches, diffuse and thunderclap like, associated with rotary vertigo, visual blur, and vomiting. The risk factors were represented by ethylism, hypertension and type II android obesity. On physical examination, the blood pressure was $170 / 100 \mathrm{mmHg}$, the consciousness was obsessed with a Glasgow score of 9/15 and the temperature at $37^{\circ} \mathrm{C}$. She exhibited motor agitation, visual hallucinations with zoopsies, and stiffness of the neck with the presence of Brudzinski and Kernig signs, and bilateral sign of Babinski. The sensitivity and coordination tests were difficult. There was photophobia with conjunctival hyperemia and isocorical pupils with retained oculomotricity. The patient presented in summary an intracranial hypertension syndrome, a meningeal syndrome, a confusional syndrome, associated to systolic and diastolic high blood pressures WHO grade II and type II obesity. All these syndromes lead to cerebrospinal hemorrhage. The biological analyzes with the electrocardiogram were normal. The brain scan revealed a spontaneous hyperdensity located in the splenium of the corpus callosum and subarachnoid spaces (Figures 2).
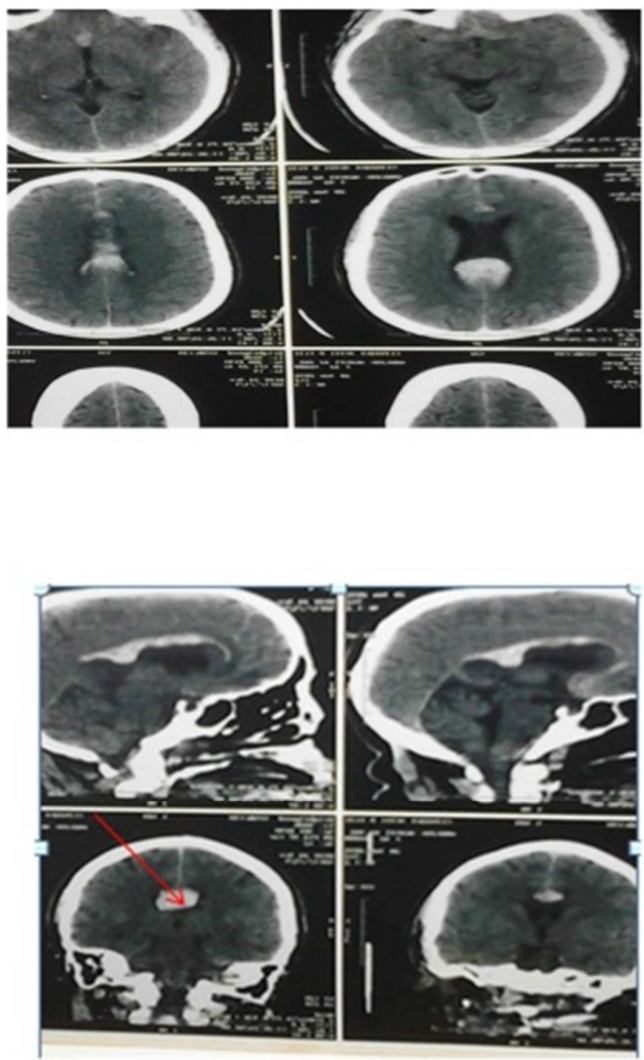

Figure 2. Patient 1: CT scan of axial, sagittal and frontal brain sections showed hyperdensity of corpus callosum and sub arachnoid hemorrhages. 
CT angiography and cerebral angioRM were not performed due to lack of financial ressources. The patient had received anti-edematous, analgesic, myorelaxant, anxiolytic and antihypertensive treatment.

The evolution was marked on day5 by persistent of meningeal signs and language disorders such as Broca aphasia with motor perseverance and improvement of the consciousness state. At one month later, we have noted the persistence of visual hallucinations, ideomotor apraxia, left unilateral digital anomia, frontal syndrome, and a modified Rankin score 1, and language recovery. The patient was seen at 3 months with persistent of slight above signs without hallucinations.

\subsection{CASE 2}

A 38-year-old patient, right-handed and reseller, was admitted to the neurology intensive cure unit for a loss of consciousness evolving two hours before admission. The unconsciousness was preceded by unusual moderate intensive headaches with periorbital irradiation, associated to morning vomiting and sonophotophobia. The observed risk factors were hypertension for 2 years and android obesity.

The admission examination noted a blood pressure of $150 / 90 \mathrm{mmHg}$ and a weight of $95 \mathrm{~kg}$ for a height of $1.68 \mathrm{~m}$. The neurological exam had noted normal consciousness, motor aphasia, Brudzinski's sign and neck stiffness, left hemiballism movement, right hemiparesis with crural predominancy, and decreased osteotendinous reflexes, and a bilateral Babinski sign. The sensitivity, cranial nerves and coordination test was normal. The clinical examination had objective intracranial hypertension syndrome, a meningeal syndrome, a left extra pyramidal syndrome (hemichoreoballism), a partial right pyramidal syndrome, a systolic hypertension of WHO grade I and android obesity. These symptoms lead to cerebromeningeal hemorrhage. The brain CT showed a spontaneous hyperdensity fusing in the splenium, trunk and genu of the corpus callosum and ventricles (Figure 3).

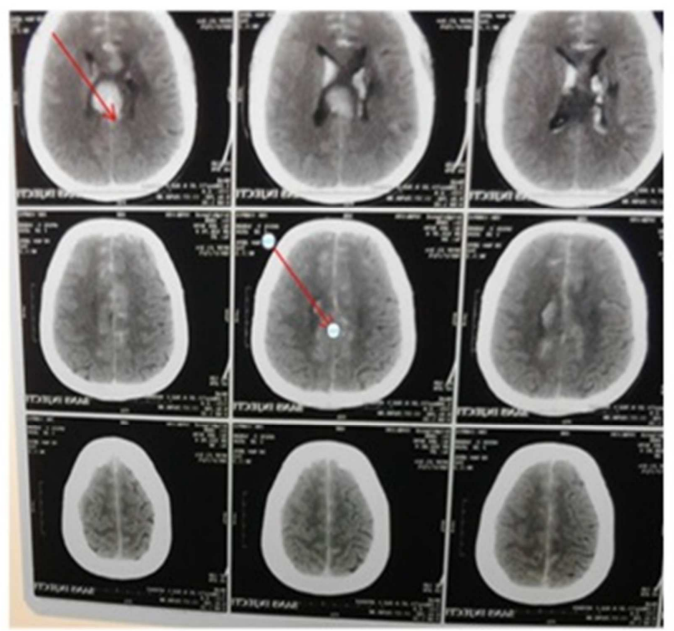

Figure 3. Patient 2: CT scan of brain, axial section at day 2 showed hyperdensity of corpus callosum splenium and sub arachnoid hemorrhage.
The patient received anti-edematous, analgesic, neuroleptic, myorelaxant, antihypertensive, antivasospasm (Nimodipine), and language therapy and motor rehabilitation. At day10 of evolution under treatment, the neurology examination noted a frontal syndrome, a dysarthria, a diagonostic apraxia, an agraphia of the left hand, a left tactile anomia and a regression of the choreo-ballic movements. At the seventeenth day of evolution, the patient had a modified Rankin score of 2 with moderate frontal syndrome. At 3 months post stroke, the modified Rankin score was rated at 1 , with and ideomotor slowness and no abnormal movements.

\subsection{CASE 3}

A 23-year-old woman, right-handed, housewife, recently delivered with dead twins, was admitted to emergency neurologic care unit for two episodes of generalized tonicclonic seizures, with no fever, on the fifth day of postpartum. The alteration of consciousness was preceded by unusual moderate intensive diffuse headaches, with periorbital irradiation, associated with easy vomiting. She had high blood pressure since two years and severe preeclampsia at the $30^{\text {th }}$ weeks of amenorrhea. The admission examination had noted a blood pressure of $150 / 90 \mathrm{mmHg}$. On the neurological exam, there was an ideomotor slowness, neck stiffness with the presence of the sign of Brudzinski, diminished osteo-tendinous reflexes, and bilateral Babinski sign. There was visuo-motor ataxia, sonophotophobia, and normal-sized isocorical pupils. Physical examination was normal for other functions and systems. In summary, we have observed meningeal syndrome, visuo-motor ataxia, encephalitic syndrome, WHO grade I systolic hypertension leading to cerebro-meningeal haemorrhage. The brain CT showed a spontaneous hyperdensity of the corpus callosum with ventricular contamination suggesting a broken hematoma of small abundance in the corpus callosum with contamination of the lateral ventricles (Figure 4).

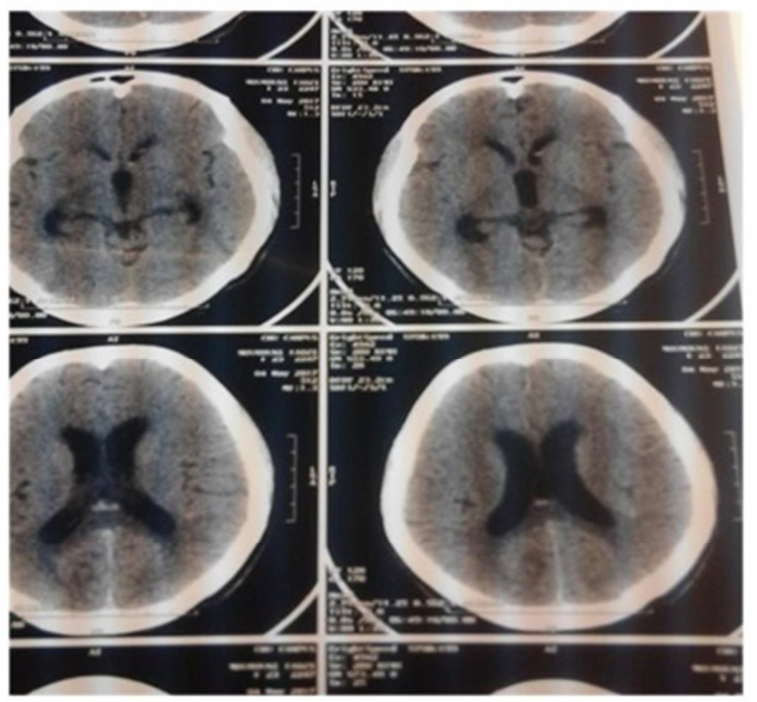

Figure 4. Patient 3: CT scan of axial brain section showed hyperdensity of splenium and meningeal spaces at day 6 . 
The patient was treated with analgesic, muscle relaxant, antihypertensive, and antivasospasm (Nimodipine). Language and motor rehabilitation was started in hospital. The evolution at the exit at day 30 was marked by a modified RANKIN score of 1, an ideomotor slowness and a motor aphasia. At 90 days post stroke, the neurological examination was strictly normal with and good language recovery.

\subsection{CASE 4}

A 51-year-old patient, right-handed, commercial, was admitted to neurology emergency care unit for progressive alteration of consciousness evolving six days before its admission. The coma was was preceded by unusual, diffuse, and thunderclap headaches associated to vomiting. She had high blood pressure poorly followed since 2 years. On admission the blood pressure was $180 / 90 \mathrm{mmHg}$. The neurological exam, had noted 7/15 of Glasgow score, a stiff neck with the presence of Brudzinski's sign, a quadriplegia with hypotonia, a four-limb osteotendinous areflexia and a bilateral Babinski sign. There was sonophotophobia, isocorical pupils with cephalic deviation to the left. In summary, we have reported an intracranial hypertension syndrome, a meningeal syndrome, a bilateral pyramidal syndrome, and WHO grade II systolic and diastolic hypertension. These syndromes lead to a diagnosis of cerebro-meningeal hemorrhage with bilateral ventricular extension. The cardiac and biological assessment was normal. The brain scan revealed spontaneous hyperdensity in the genu and trunk of the corpus callosum with lateral ventricles contamination and triventricular hydrocephalus (Figure 5).

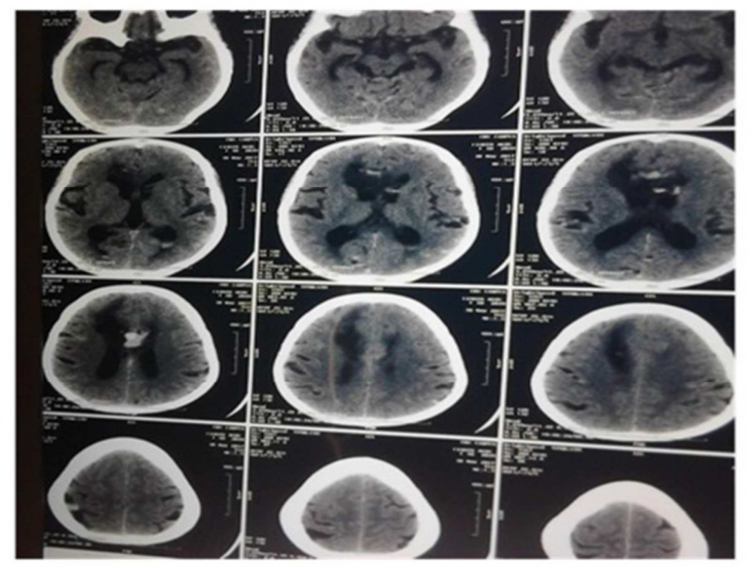

Figure 5. Patiet 4: CT scan of axial brain section showed hyperdensity of kneel and tronc of corpus callosum.

The patient had received analgesic, muscle relaxant and antihypertensive drugs, and antivasospasm (Nimodipine) treatment. Language therapy and motor rehabilitation was started during hospitalization. On 30 days evolution, the patient had a modified Rankin score of 3 with paraparesis associated to Alien hand syndrome. The CT scan control had noted a complete disappearance of cerebral haemorrhage signs. At three months, the modified RANKIN score was evaluated at 2 with ideomotor slowness.

\subsection{CASE 5}

A 50-year-old patient, right-handed, admitted to neurology emergency care unit for a sudden right motor deficit associated with a loss of consciousness, two hours before admission. This deficit was preceded by twenty-four hours of unusual moderate intensive cephalalgia of with periorbital irradiation and vomiting. He had a badly hypertension followed up since 2 years and and android obesity. The admission examination noted a blood pressure of 150/90 $\mathrm{mmHg}$. The neurological examination revealed dysarthria, ideomotor slowness, motor and verbal perseverance, partial right hemiparesis with bilateral Babinski sign, retrograde memory disorders, decreased osteotendinous reflexes, and sphincteric dyscomfort. There was sonophotophobia, isocorical pupils and stiffness of the neck with presence of the sign of Brudzinski.

In summary, we have observed a intracranial hypertension syndrome, a frontal syndrome, a meningeal syndrome, a right partial pyramidal syndrome, and a systolic and diastolic hypertension grade I of WHO and an android obesity related to cerebral haemorrhage.

The brain CT showed spontaneous hyperdensity in the genu and trunk of the corpus callosum and in ventricles suggestive of hematoma of the corpus callosum with ventricular contamination (Figure 6).

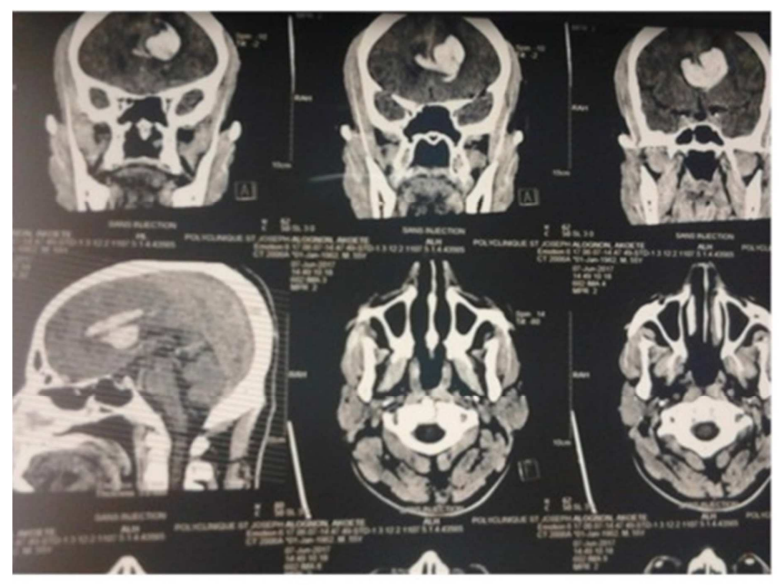

Figure 6. Patiet 5: CT scan of brain axial, coronal and sagittal sections showed hyperdensity of corpus callosum.

The patient was treated with analgesic, muscle relaxant, antihypertensive, and antivasospasms (Nimodipine). Speech therapy and motor rehabilitation was started during hospitalization. The discharge was made on the fifteenth day. The patient had motor slowness, dynamic apraxia and motor impertinence, and a modified Rankin score of 2 . The table 1 showed the recapitulative characteristics of the five cases. 
Table 1. Clinical, radiological and evolutive aspects of patients with corpus callosum hemorrhage.

\begin{tabular}{|c|c|c|c|c|c|c|c|}
\hline $\mathbf{N}^{\circ}$ & Age & Sex & Clinical signs & CT scan aspects & Evolution & $\begin{array}{l}\text { Lengh of } \\
\text { stay (d) }\end{array}$ & $\begin{array}{l}\text { Complications }>90 \\
\text { days }\end{array}$ \\
\hline 1 & 42 & $\mathrm{~F}$ & $\begin{array}{l}\text { Encephalitic syndrome } \\
\text { Meningeal syndrome }\end{array}$ & $\begin{array}{l}\text { Hyperdensity of splenium } \\
\text { and meningeal spaces }\end{array}$ & Favorable & 10 & $\begin{array}{l}\text { Frontal syndrome } \\
\text { Ideomotive apraxia }\end{array}$ \\
\hline 2 & 38 & $\mathrm{~F}$ & $\begin{array}{l}\text { Encephalitic syndrome } \\
\text { Meningeal syndrome } \\
\text { Hemi ballic and choreic movement disorders } \\
\text { Left partial pyramidal syndrome } \\
\text { Hemispheric disconnection syndrome }\end{array}$ & $\begin{array}{l}\text { Hyperdensity of splenium, } \\
\text { tronc and genu of CC with } \\
\text { ventriculary contamination }\end{array}$ & Favorable & 15 & $\begin{array}{l}\text { Frontal syndrome } \\
\text { Ideomotive } \\
\text { slowness }\end{array}$ \\
\hline 3 & 23 & $\mathrm{~F}$ & $\begin{array}{l}\text { Encephalitic syndrome } \\
\text { Meningeal syndrome } \\
\text { Sensory frontal syndrome }\end{array}$ & $\begin{array}{l}\text { Hyperdensity of splenium } \\
\text { and meningeal spaces }\end{array}$ & Favorable & 30 & Normal \\
\hline 4 & 51 & $\mathrm{~F}$ & $\begin{array}{l}\text { Intracranial hypertension syndrome, } \\
\text { Meningeal syndrome, } \\
\text { Bilateral pyramidal syndrome }\end{array}$ & $\begin{array}{l}\text { Hyperdensity of genu and } \\
\text { tronc of CC, lateral } \\
\text { ventricules contamination, } \\
\text { triventriculary hydrocephalus }\end{array}$ & Favorable & 21 & $\begin{array}{l}\text { Paraparesia, } \\
\text { Alien hand } \\
\text { syndrome }\end{array}$ \\
\hline 5 & 50 & M & $\begin{array}{l}\text { Intracranial hypertension syndrome, } \\
\text { Meningeal syndrome, } \\
\text { Bilateral partial pyramidal syndrome Frontal } \\
\text { syndrome }\end{array}$ & $\begin{array}{l}\text { Hyperdensity of genu and } \\
\text { tronc of CC and ventricules }\end{array}$ & Favorable & 15 & $\begin{array}{l}\text { Ideomotive } \\
\text { slowness, Dynamic } \\
\text { apraxia, Motor } \\
\text { impertinence }\end{array}$ \\
\hline
\end{tabular}

Legend: M: Male; F: Female; CT: Computer Tomography; d: day; No: order number; CC: Corpus Callosum.

\section{Discussion}

\subsection{Methodology}

The study had reported five cases of hemorrhagic strokes located in the corpus callosun. There are some limitations in this study to be noted. It concerns in firsthand the selection bias related to some missing patients who could not afford the clinical facilities for many reasons and stayed with heavy sequels or those who may die without diagnosis within transport or attending traditional healers. Secondly, lesions of the corpus callosum did not have complete correspondence with clinical symptoms. The third hand of bias concerns the lack of financial resources of the patients and the insufficiency of the technical platform in our hospital structures, which unable to undergo brain imaging. These deficiencies reduce the practice of useful explorations for the etiological diagnosis including the complete assessment of hemostasis, cerebral fMRI, cerebral angio-MRI and diffusion-tensor imaging for possible complications. The findings of the observational study may not be generalized to the whole country population and further studies need to be performed to collect these misdiagnosis cases. However, the specific of the cases and its rich symptoms need to be shared with the widely scientific comminutees.

\subsection{Epidemiology}

During the last three years, 3,922 patients have been hospitalized in the neurology department of the hospital. Approximately 2,355 patients had a stroke, 915 of which were hemorrhagic. These results are similar to previous reported study $[5,7]$. The study had reported five cases of corpus callosum haematomas with a frequency of $0.3 \%$ of all strokes, and overall stroke frequency of $38.2 \%[6,8]$. This extreme rarity of the callosum hematoma is explained by the configuration of the perforating branches that would protect the corpus callosum from cerebral hemorrhages. The corpus callosum is a small anatomical structure with anterior localization. Hematomas often include several anatomical structures and it is difficult to identify the elements directly involved.

Complete section of the corpus callosum disrupts a massive component of interhemispheric anatomical connectivity. In the present study, the diagnosis was made with clinical exam and performing brain CT scan. This study is important because of the opportunity it offers to examine the relationship between the brain's connectional anatomies and to meet the dysconnection syndrome with its rich semiology. It is impossible to perform fMRI signal to identify differents functionnal areas of the $\mathrm{CC}$ and the principle origin of the heamorrahge. More recent work using resting functional connectivity MRI in humans has demonstrated distinct pathways for the head and body of the CC and suggests that the splenium may be an important mediator of interhemispheric transfer pathway $[9,12]$.

The average age was 33.4 years with extremes of 23 and 51 years old. This result is in accordance with those found in the literature which states that hematomas occur more in young subjects and all haemorrhages of the corpus callosum in young adults $[8,11]$. The female predominance is similar to some previous studies but in controverse with other past works $[5,8]$. The sex is not a significative indicator in CC haemorrhage.

\subsection{Clinical Examination}

In the acute phase, disturbances of consciousness and signs of intracranial hypertension were prominent in the five reported cases. These results are similar to those reported in the literature which concluded that hematomas in their unusual location would result in less hemiplegia than in its conventional locations and especially if they do not destroy functional motor areas. On the other hand, the deterioration of the consciousness can settle more quickly in the rare 
localizations due to the mass effect induced by the volume of the hematoma and the classical perilesional edema [3, 7]. Losses in transcallosal motor pathways following unilateral brain injuries can contribute to deficits with bimanual coordination, complex unilateral tasks, and locomotion [1, 3]. Based on the results, the corpus callosum is affected in greater extent by haemorragic disruption compared to unilateral injuries occurring early in life in agenesis defect, infection or infarction diseases. Differences between pediatric and adult hemiplegia in $\mathrm{CC}$ injuries may be due to the developmental and myelination state of the corpus callosum fibers at the time of injury leading to neural reorganization $[13,14]$.

The structure of CC cell and its connecting pathways were suffering from the haematoma volume pression but after resorption, the nerves impulses retake its connections that explain the regression of the dysconnection signs with more recovery and normalization of activity compare to simple or complex CC injury attacks $[4,7,12]$.

The most common causes of reversible focal lesions of the SCC are viral encephalitis, antiepileptic drug toxicity and hypoglycemic encephalopathy. Many other causes have been reported, including traumatic axonal injury and haemorhagic fevers [13]. The mechanisms that underlie haemorragic injuries of the CC are poorly understood, and those that have been proposed are based mostly on autopsy findings. Some researchers reported that massive hematomas were produced by severe head injury or vessels disease damage, persistent hemodynamic stress, cavernous angioma, aneurysms or hemorrhagic fever and vessels disease in the moyamoya [8].

In the present cases, the main causes must be the high blood pressure and chronic alcohol drinking. Therefore, intracerebral hemorrhage in the splenium of corpus callosum was considered quite rare but most frequent haemorragic seats in these reported cases [9]. Moreover, there was no similar results find in the literature, the actual pathophysiological mechanism seems unclear, especially since the patients did not have access to other means of brain imaging to search the main etiologies $[6,9]$. Thus, additional studies are needed to explain these anatomical correlations using functional MRI or radiological methods.

\subsection{Neuropsychological Disorders}

Different neuropsychological pictures were observed. In the first two cases reported, the patients had presented with behavioral disorders, phasic and dyspraxic symptoms, digital anomia on the left with Allen Hand Syndrome. Moreover, the third reported case had presented only ideomotor slowness signs. These data are similar to the cases reported in the previous studies $[13,15]$. But the second case reported had presented in addition to the signs rosen above, abnormal movements in the right hemi-body. The relationship between corpus callosum and behavior is well known. Many authors had reported neuropsychological disorders due to lesions involving $\mathrm{CC}$ connective pathways $[6,7,9]$. Other psychiatric symptoms were repoorted such as dementia, depression, schizophrenia, and psychosis [8,
$11,14]$. Usually the haematomas are so extensive that it is not easy to assess whether the primary origin of symptoms is the damage of corpus callosum or the involvement of other adjacent structures. However, in rare cases, psychiatric symptoms are associated with a wellcircumscribed lesion of the corpus callosum [8, 13]. As mentioned before, the most common clinical findings in patients with $\mathrm{CC}$ hemorrhage described in the literature are mental retardation, visual problems, speech delay, seizures, abnormal muscular tone, neglect syndrome and feeding problems [9, 10]. None of the fives reported patients had presented these symptoms as they were adult with normal formed $\mathrm{CC}$ that damage by the haematoma.

\subsection{Seat of the Hematoma and Volume of the Hematoma}

The hematoma seats interested in order of frequency were constituted of the splenium with $60 \%$ of cases, the genu $20 \%$ and trunk of the corpus callosum in $20 \%$. This result is similar to that reported in the literature which found that focal lesions during vascular pathologies of the corpus callosum affected preferentially the splenium [1, 3]. This high incidence of splenium injuried is linked to its double vascularization, particularly with the posterior cerebral artery, which would expose it little more than the other parts of the corpus callosum $[3,8]$.

Conserning the risk factors, studies have shown that there is a link between the level of high blood pressure and the occuring of stroke, regardless of type and location [12, 13]. The hypertension drive is the leading cause of deep hemispheral and infratentorial haemorrhages, with varying numbers [10]. It is recognized that regular alcohol consumption in excess of $60 \mathrm{~g} /$ day is associated with an increased risk of stroke compared to abstinent subjects [11, 12]. The high frequency of alcohol, noted in this work (80\%) is consistent with the literature findings. Alcoholism is the second risk factor in atypical topography hematomas, with a frequency of $20 \%$. The literature had reported that the relative risk of occurrence of a hematoma in an alcoholic subject is $2.2[10,12]$.

\subsection{Evolution Under Treatment and Prognostic Factors}

No cases of death were recorded during the work. This result differs from that reported in previous study where they had observed a mortality in one out of two cases during the first week of hospitalization. This discrepancy could be justified on the one hand, by the high volume of hematomas with triventricular hemorrhagic flood, the advanced age of the patients and on the other hand by the difficulties of management. On the CT scan, the hematomas were of low volumes, with average of $17.2 \mathrm{ml}$ and a mean delay of 3-day before performing brain scan. According to some authors, the effectiveness of stroke management reflects a country's health system capabilities $[12,13]$. We have noted on the initial CT scan, the presence of ventricular contamination in $80 \%$ of cases, but the average Glasgow scores were at $11 / 15$, and a small volume of hematomas. This result corroborates 
with the previous work who reported that the poor prognostic factors are related to large hemorrhagic volume, a low Glasgow score, and the presence of ventricular hemorrhage on the initial scan $[14,15]$.

\section{Conclusion}

The corpus callosum appears to be an extremely rare seat of cerebral hemorrhage. This location of the hematoma gives a serious affection with rather misleading semiology. The neuropsychological disorders were in the foreground. Splenium was the most affected part. The dominant risk factors were high blood pressure and alcoholism. The outcome was favorable with slight complications. The $\mathrm{CC}$ is essential in sensory experience since it transfers information from one hemisphere to another. In a CC dysfunction, each hemisphere becomes isolated, acting as two separate brains. Although general intelligence and behavior appear normal, other functions are affected. Even though the parietal lobe is the one classical related with the neglect syndrome, other structures are involved in its anatomy, as the CC.

\section{References}

[1] Raybaud C. The corpus callosum, the other great forebrain commissures, and the septum pellucidum: Anatomy, development, and malformation. Neuroradiology. 2010; 52: $447-51$.

[2] Knyazeva MG. Splenium of corpus callosum: Patterns of interhemispheric interaction in children and adults. Neural Plast 2013; 6: 39-43.

[3] Fenlon LR, Richards LJ. Contralateral targeting of the corpus callosum in normal and pathological brain function. Trends Neurosci. 2015; 38: 264-72.

[4] Tovar-Moll F, Monteiro M, Andrade J, Bramati IE, ViannaBarbosa R, Marins T et al. Structural and functional brain rewiring clarifies preserved interhemispheric transfer in humans born without the corpus callosum. Proc Natl Acad Sci US A. 2014; 111: 7843-8.

[5] In Hwan K, Lee S, Lee Ch-Y, Lee DG. Intracranial Hemorrhage in the Corpus Callosum Presenting as Callosal
Disconnection Syndrome: FDG-PET and Tractography: A Case Report. Ann Rehabil Med 2014; 38 (6): 871-5.

[6] Funatsu N, Hayakawa M, Tokuda N, Toyoda K. Transient Prosopometamorphopsia Restricted to the Left Eye Caused by Ischemia at the Right Splenium of the Corpus Callosum. Intern Med 2017; 56: 2933-5.

[7] Nagaishi A, Narita T, Gondo Y, Nakane S, Fukudome T, Matsuo H. Left-sided metamorphopsia of the face and simple objects caused by an infarction at the right side of the splenium of the corpus callosum. Rinsho Shinkeigaku (Clin Neurol) 2015; 55: 465-71.

[8] Panciani PP, Roca E, Lodoli G, Fontanella MM, Maldini AE, Panciani PP et al. Sudden worsening after subdural haematoma surgery: will there be a corpus callosum injury? BMJ Case Rep 2014; 36: 2013-8.

[9] Gao X, Li B, Chu W, Sun X, Sun Ch. Alien hand syndrome following corpus callosum infarction: A case report and review of the literature. Experimental And Therapeutic Medicine 2016; 12: 2129-35.

[10] Pavesi G, Causin F, Feletti A. Cavernous Angioma of the Corpus Callosum Presenting with Acute Psychosis. Behavioural Neurology 2014; 2: 1-4.

[11] Li S, Sun X, Bai YM, Qin HM, Wu XM, Zhang X et al. Infarction of the corpus callosum: A retrospective clinical investigation. PLoS One. 2015; 10: e0120409.

[12] Gomes D, Fonseca M, Garrotes M, Lima MR, Mendonça M, Pereira $\mathrm{M}$ et al. Callosum and Neglect Syndrome: Clinical Findings After Meningioma Removal and Anatomical Review. J Neurosci Rural Pract. 2017; 8 (1): 101-6.

[13] Dragone A, Lasaponara S, Silvetti M, Macaluso E, Doricchi F. Selective reorienting response of the left hemisphere to invalid visual targets in the right side of space: Relevance for the spatial neglect syndrome. Cortex. 2015; 65: 31-5.

[14] Kim DS, Choi HJ, Yang JS, Cho YJ, Kang SH. Radiologic Determination of Corpus Callosum Injury in Patients with Mild Traumatic Brain Injury and Associated Clinical Characteristics. J Korean Neurosurg Soc 2015; 58 (2): 131-6.

[15] Ridley B, Beltramone M, Wirsich J, Le Troter A, Tramoni E, Aubert $\mathrm{S}$ et al. Alien hand, restless brain: Salience network and interhemispheric connectivity disruption parallel emergence and extinction of diagonistic dyspraxia. Front Hum Neurosci. 2016; 10: 307-11. 